\title{
A Revised Distributed Interpretation for Ontology Integration
}

\author{
Kun Liu*, ${ }^{2}$, Liang Zhang ${ }^{2}$, Shengqun Tang ${ }^{2}$, Xue Qin ${ }^{2}$ \\ * State Key Lab of Software Engineering, Wuhan University \\ Wuhan, China \\ ${ }^{2}$ Department of Computer Science, Hubei University of Economics \\ Wuhan, China \\ lovecpp@sohu.com
}

\begin{abstract}
Under the background of information integration, the semantics of ontology integration is still an open issue. In this paper, we propose a revised distributed interpretation which is adapted from distributed description logics. In our proposal, ontology integration is taken as global ontology and local ontologies connected by ontology mapping. They are respectively interpreted with DL semantics and Semantic Import semantics. In this way, our method can facilitate understanding and maintenance of ontology integration.
\end{abstract}

Keywords-Ontology integration, Ontology mapping, Interpretation

\section{INTRODUCTION}

Ontology has played a great role in resolving the heterogeneous of information[14][5][6]. Ontologies are linked and integrated together in order to communication among information sources. However how to interpret ontology integration is an open issue.

Borgida and Serafini[3][4] presented DDL(Distributed Description Logics) to describe distributed ontologies. But axioms across ontologies are not allowed which is not convenient for ontology integration. Fahad[7] talked about semantics of merged ontologies. However, according to [8], there are some differences between ontology merge and ontology integration. So [7] is not well suitable for ontology integration.

In this paper, we propose an interpretation based on distributed interpretation which has been discussed in DDL. We introduce some ideas of semantic import[11] to revise bridge rules of DDL and give rigorous definition to revision Then we talk about model-theoretic of revised distributed interpretation. At last we make some comparison between ours and other methods.

The rest of paper is organized as follows: Section II explains the motivation of our method. Section III talks about some preliminary knowledge. Section IV introduces our proposed method. In section $\mathrm{V}$, we make some discussion with our method. Section VI introduces some related work. We make conclusions in section VII.

\section{MOTIVATION}

In [2], Borgida and Serafini proposed DDL to describe integration information system(IS). Each IS is described with DL, and then relations between each IS are turned into bridge rules between DL ontologies. Flow of information from one
IS to another IS can also be described by bridge rules. This situation is applied to information integration. But bridge rules employ correspondence to describe relations of concepts or roles between two DL ontologies. It is not DL tradition which uses subsumption expressions or something else. And more, limited to bridge rules, DDL takes one ontology as another one's context, so its reasoning uses context reasoning method[11][13][14]. In the case of information integration, query answering is usually handled at global side and translate query into queries over local ends. Context reasoning is not well suited to query decomposition.

In fact, global ontology and local ontologies in information integration have relations and should not be separated. This means some concepts or roles have direct relations between global ontology and local ontologies. It is similar with some cases of ontology reuse, but ontologies on each side do not include any syntactic symbols from the other side. This inspires us to introduce semantic import[11] to improve this situation.

\section{PRELIMINARY}

From the DDL point of view, multiple ontologies are interconnected by semantic mapping. DDL represents semantic mapping with bridge rules which comprise three types of mapping rules.

Into rule: $\mathrm{i}: \mathrm{C} \stackrel{\mathrm{v}}{\longrightarrow} j: D$

Onto rule: $\mathrm{i}: \mathrm{C} \longmapsto j: D$

Equivalent rule: $\mathrm{i}: \mathrm{C} \stackrel{\equiv}{\longrightarrow}: D$

Based on bridge rules, DDL can formalize a pair of ontologies linked by semantic mapping.

Definition 1(Distributed T-Box): A distributed T-Box $\mathfrak{T}=<\left\{T_{\mathrm{i}}\right\}_{\mathrm{i} \in I},\left\{\mathfrak{B}_{\mathrm{ij}}\right\}_{\mathrm{i} \neq \mathrm{j} \in \mathrm{I}}>$. The set of indices $\mathrm{I}$ is defined as $\{1,2\} . T_{i}$ denotes $T$-Box ontology which includes concepts , roles and axioms. The bridge rules constructing semantic relations from $T_{i}$ to $T_{j}$ are denoted by $\mathfrak{B}_{\mathrm{ij}}$. Each rule in $\mathfrak{B}_{\mathrm{ij}}$ has a certain type, such as into ,onto or equivalent rule.

DDL establishes distributed interpretation $\mathfrak{I}$ for $\mathfrak{T}$. It consists of two elements. One is for $T_{j}$. The other is for $\mathfrak{B}_{\mathrm{ij}}$. For $\mathrm{T}_{\mathrm{i}}$, DDL adopt model-theoretic semantics of DL which called local interpretation $I_{i}$ in DDL. The semantics of $\mathfrak{B}_{\mathrm{ij}}$ is based on a domain relation $r_{\mathrm{ij}}=4^{\mathrm{I} i} \times 4^{\mathrm{Ij}}$ that connects 
interpretation domains of $\mathrm{T}_{\mathrm{i}}$ and $\mathrm{T}_{\mathrm{j}} . r_{\mathrm{ij}}(x)$ is used to denote $\left\{y \in \Delta^{\mathrm{I}_{j}} \mid(x, y) \in r_{i j}\right\}$.

Definition 2 (Distributed interpretation): A distributed interpretation $\mathfrak{I}$ for $\mathfrak{T}$ is defined as a pair of elements $\mathfrak{I}=$ $<\left\{\left\{\mathrm{I}_{\mathrm{i}}\right\} i \in I,\left\{r_{\mathrm{ij}}\right\} i, j \in I \mathrm{~B}_{\mathrm{B}}\right\}>$.

Based on distributed interpretation, DDL defines satisfiability and consistency.

Defintion 3 (Satisfiability): The distributed interpretation $\mathfrak{I}=<\left\{\left\{\mathrm{I}_{\mathrm{i}}\right\} i \in I,\left\{r_{\mathrm{ij}}\right\} i, j \in I\right\}>$ satisfies a distributed $\mathrm{T}$ Box if and only if:

(1) $I_{i}$ satisfies $T_{i}$;

(2) for every bridge rule in $\mathfrak{B}_{\mathrm{ij}}$ :

$r_{\mathrm{ij}}\left(\mathrm{C}^{\mathrm{I} i}\right) \subseteq \mathrm{D}^{\mathrm{Ij}}$, for each into rule $\mathrm{i}: \mathrm{C} \stackrel{\mathrm{v}}{\longrightarrow} j: D$

$r_{\mathrm{ij}}\left(\mathrm{C}^{\mathrm{I} i}\right) \supseteq \mathrm{D}^{\mathrm{I} j}$, for each onto rule $\mathrm{i}: \mathrm{C} \longmapsto j: D$

$r_{\mathrm{ij}}\left(\mathrm{C}^{\mathrm{I} i}\right)=\mathrm{D}^{\mathrm{Ij}}$, for each equivalent rule $\mathrm{i}: \mathrm{C} \stackrel{\equiv}{\longrightarrow}: D$

Consistency in DDL is reduced to check whether a local concept of bridge rules is satisfiable or not. If all local concepts are satisfied by local interpretation $\mathrm{I}_{\mathrm{i}}$ and bridge rules interptretation $r_{\mathrm{ij}}$, the distributed T-Box is consistent.

\section{OUR PROPOSED APPROACH}

\section{A. Global and Local ontolgies}

In our approach, we adopt hybrid ontologies to describe information integration[1], but we make a little modification. This method use local ontologies to represent information sources and global ontology to integrate all local ontologies, as showed in firgure1.

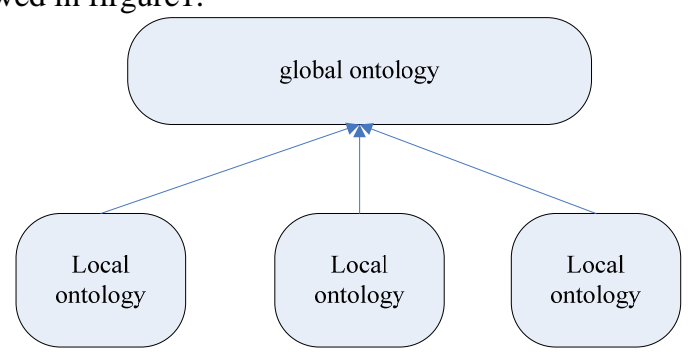

Figure 1 hybrid ontologies

There are mappings between global ontology and local ontologies which relate concept or roles. But different from Wache[14], there no relations among local ontologies. Then global ontology can serve as bridges or connections between local ontologies.

Hybrid ontologies can also be denoted by $\mathfrak{T}^{\prime}=$ $<\mathrm{T}_{\mathrm{g}},\left\{\mathrm{T}_{\mathrm{i}}\right\}_{\mathrm{i} \in I},\left\{\mathfrak{B}_{\mathrm{ig}}\right\}_{\mathrm{i} \in \mathrm{I}}>$. $\mathrm{T}_{\mathrm{g}}$ means global ontology and $\mathrm{T}_{\mathrm{i}}$ represent each local ontology. $\mathfrak{B}_{\text {ig }}$ shows that a local ontology $\mathrm{T}_{\mathrm{i}}$ has mappings with global ontology which will be introduced in the following sections.

\section{B. Concept or role mapping}

Globale ontology and local ontologies have relation through concepts or roles. In DDL, their relation are represented by bridge rules. We choose mapping which is similar to bridge rules in DDL to represent relations between concepts coming from ontologies.

From a syntactic point of view, mappings do not appear on global or local side. But it is not same as linking axioms like $\varepsilon$-connections[8].

Definition 4(concepts mapping): Concepts mapping from ontology $T_{i}$ to $T_{j}$ is denoted by $\mathfrak{b}_{i j}$ which includes three types of relation:

i) equation: $\mathrm{i}: \mathrm{C} \stackrel{\equiv}{\longrightarrow} \mathrm{D} D$

ii) into: $\mathrm{i}: \mathrm{C} \stackrel{\mathrm{v}}{\longrightarrow} j: D$

iii) disjointness: $\mathrm{i}: \mathrm{C} \stackrel{\perp}{\longrightarrow} j: D$ 5.

Similar, definition of roles mapping is given in definition

Defintion 5(roles mapping): Role mapping from ontology $T_{i}$ to $T_{j}$ is denoted by $\mathfrak{b}_{i j}$ which includes two types of relation:

i) equation: $i: R \equiv j: S$

ii) into: $i: R \sqsubseteq j: S$

The notion $\rightarrow$ is borrowed from DDL. Some ontology matching tools can help to find equation or into relation between concepts.

Example1 : there are two ontologies. One is University and the other is UNIV. They have the following concept and role mappings.

\section{University: Professor $\stackrel{\sqsubseteq}{\longrightarrow} U N I V:$ Professor \\ University: Course $\stackrel{\sqsubseteq}{\longrightarrow}$ UNIV : Course \\ University: teach $\sqsubseteq U N I V:$ teacherOf}

In this example, Professor and Course in University has a corresponding concept in UNIV and teach is mapped into teacherOf in UNIV.

\section{Interpretation}

In order to give a distributed interpretation, ontologies which are involved in concepts or roles mapping should take mapping relations into account. It comprises two ways:

1) Mapping relations as imported axioms to $T_{j}$

In the situation of information integration, global ontology is the center and its semantics is the most important. All other local ontologies's semantics should conform to it.

For ontology integration, mapping relations point from local ontologies to global ontology. Under this situation, the semantics of global ontology should maintain and its consistency should not violate. So not all mapping relations are accepted by $\mathrm{T}_{\mathrm{j}}$. Three types of relations can be seen as the following axioms:

i) $i: C \equiv j: D$

ii) $i: C \sqsubseteq j: D$

iii) $i: C$ u $j: D \sqsubseteq$ ?

But iii) is not certain in all ontology integration.

In this situation, cross ontology expressions are allowed. For example, i:C can directly used in axioms i), just like $C \equiv D$ in an ontology. 
2) Mapping relations as ontology reuse for $T_{i}$

In descriping mappings with bridge rules, it concertrates on express . Based on this idea, from the point view of ontology $T_{j}$, concepts or roles defined in ontology $T_{j}$ can be seen ontology reuse. It means ontology $\mathrm{T}_{\mathrm{j}}$ reuse some concepts or roles from ontology $\mathrm{T}_{\mathrm{j}}$. And more, under the background of ontology integration, these reused concepts or roles should maitain their semantic in ontology $T_{j}$ when it serves as global ontology.

For each $\mathrm{T}_{\mathrm{i}}$, an interpretation $\mathrm{I}_{\mathrm{i}}$ should consider two aspects[5]:

1) if $\mathrm{j}: \mathrm{D}$ is a class name in $\mathrm{T}_{\mathrm{j}}$, then $(j: D)^{\mathrm{I} i}=$ $(j: D)^{\mathrm{Ij}} \cap 4^{\mathrm{I} i}$.

2) if $j: R$ is a role name in $T_{j}$, then for all $d \in$ $4^{\mathrm{I} i} \cap 4^{\mathrm{I} j}$, for all $\mathrm{d}^{\prime} \in 4^{\mathrm{I} j},<\mathrm{d}, \mathrm{d}^{\prime}>\in(j: R)^{\mathrm{I} i}$, iff $<\mathrm{d}$, $\mathrm{d}^{\prime}>\in(j: R)^{\mathrm{I} j}$

\section{Consistency}

Definition6 (Satisfiability): A distributed interpretation $\mathfrak{I}^{\prime}$ $=<\left\{\left\{\mathrm{I}_{\mathrm{i}}^{\prime}\right\} i \in I, \mathrm{Ig}_{\mathrm{g}}\right\}$ satisfies $\mathfrak{T}^{\prime}$, when the following condition are satisfied:

1) $\mathfrak{I}^{\prime} \vDash \mathrm{T}_{\mathrm{g}} \quad\left[\left\{\mathfrak{B}_{\mathrm{ig}}\right\}_{\mathrm{i} \in \mathrm{I}}\right.$, if $\mathrm{I}_{\mathrm{g}} \vDash \mathrm{T}_{\mathrm{g}}$ and $\mathrm{I}_{\mathrm{g}} \vDash$ $\left\{\mathfrak{B}_{\mathrm{ig}}\right\}_{\mathrm{i} \in \mathrm{I}}$;

2) $\mathfrak{I}^{\prime} \vDash T_{i}$, if $I^{\prime}{ }_{i} \vDash T_{i}$;

3) for every concept axiom in $\mathfrak{B}_{\text {ig }}$, $\mathfrak{I}^{\prime} \vDash i: C \equiv j: D$, if $(\mathrm{i}: \mathrm{C}){ }^{\mathrm{I}} \mathrm{i} \equiv(\mathrm{j}: \mathrm{D}){ }^{\mathrm{I}} \mathrm{i}$; $\mathfrak{I}^{\prime} \vDash i: C \sqsubseteq j: D$,if (i:C) ${ }^{\mathrm{I}} \mathrm{i} \subset(\mathrm{j}: \mathrm{D}){ }^{\mathrm{I}} \mathrm{i}$; $\mathfrak{I}^{\prime} \vDash i: C \mathrm{u} j: D \sqsubseteq$ ? , if (i:C) ${ }^{\mathrm{I}} \mathrm{i} \cap(\mathrm{j}: \mathrm{D}){ }^{\mathrm{I}} \mathrm{i}=\varnothing$;

4) for every role axiom in $\mathfrak{B}_{\mathrm{ig}}$

$$
\begin{aligned}
& \mathfrak{I}^{\prime} \vDash i: R \equiv j: S \text {, if (i:R) }{ }^{\mathrm{I}} \mathrm{i} \equiv(\mathrm{j}: \mathrm{S}) \mathrm{I}^{\prime} \mathrm{i} \\
& \mathfrak{I}^{\prime} \vDash i: R \sqsubseteq j: S \text {, if (i:R) }{ }^{\mathrm{I}} \mathrm{i} \sqsubseteq(\mathrm{j}: \mathrm{S}){ }^{\mathrm{I}} \mathrm{i}
\end{aligned}
$$

This interpretation is also called a model of $\mathfrak{T}^{\prime}$.

$I^{\prime} i$ in this definition is not same as the $I_{i}$ in $\mathfrak{I}^{\prime}$. One is that I'i should not only satisfy the corresponding local ontology $\mathrm{T}_{\mathrm{i}}$, but also satisfy the concepts or roles reused from global ontology. $\mathrm{T}_{\mathrm{g}}$, as showed by condition 3 .

Based on the definition of satisfiability, consistency is given in definition 7 .

Definition 7 (Consistency): for an ontology integration $\mathfrak{T}^{\prime}$, if a model can be found to satisfied $\mathfrak{T}^{\prime}$, then $\mathfrak{T}^{\prime}$ is consistent, or it is inconsistent.

\section{E. Consistency checking}

According to definition of consistency, consistency checking is divided into two processes as showed in figure 2. One is for $T_{g}\left[\left\{\mathfrak{B}_{\mathrm{ig}}\right\}_{\mathrm{i} \in \mathrm{I}}\right.$, and the other is for every combination of $\mathrm{T}_{\mathrm{i}}$ and $\mathfrak{B}_{\mathrm{ig}}$.

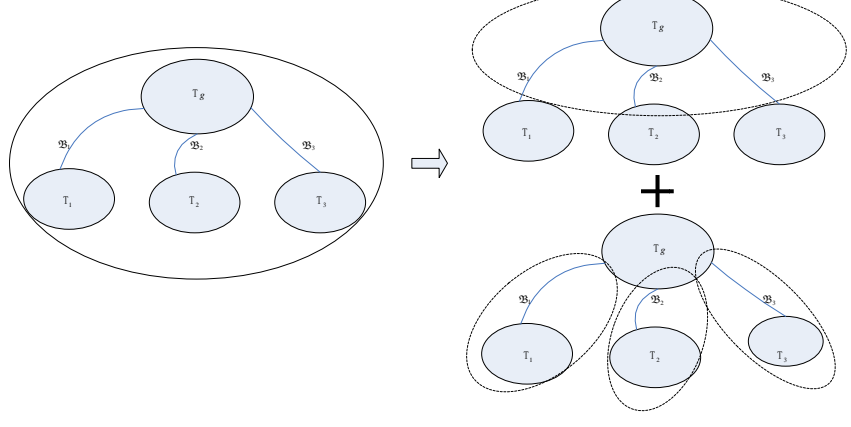

Figure 2 consistency checking

Firstly, it is a simple task to check $\mathrm{T}_{\mathrm{g}}\left[\left\{\mathfrak{B}_{\mathrm{ig}}\right\}_{\mathrm{i} \in \mathrm{I}}\right.$. Because in this process, $\mathfrak{B}_{\text {ig }}$ is seen as added axioms to $T_{g}$. The semantics of $\mathfrak{B}_{\text {ig }}$ should conform to $\mathrm{T}_{\mathrm{g}}$.

Secondly, for $\mathfrak{B}_{\mathrm{ig}}$ has been checked in $\mathrm{T}_{\mathrm{g}}$, the concepts or roles used in $\mathfrak{B}_{\mathrm{ig}}$ become reused knowledge in each $T_{i}$. So each combination of $T_{i}$ and $\mathfrak{B}_{\text {ig }}$ needs to be tested.

In the future, we will develop the corresponding algorithms about how to check consistency.

\section{DISCUSSION}

Global interpretation is a nature way to explain ontology integration which means that integrated ontologies are seen as a whole ontology. In this way, many existing methods and tools can be reused. But, if there are so many heterogeneous ontologies and so much dissimilarity, it is a hard work to check consistency and repair inconsistency, especially when the amount of ontologies reaches a degree.

Compared to global interpretation, distributed interpretation has some advantages. It conforms to the status of scattering ontologies. Though DDL adopts distributed interpretation, it has some limits for ontology integration, such as, cross concept inclusions are not allowed. For ontology integration all ontologies should be combined together which is most different from DDL.

Ontology mapping connects distributed ontologies and its semantics is the sole of distributed interpretation. In DDL, it doesn't consider the need of combination. In our method, semantic import is adopted to revise distributed interpretation of DDL. And roles subsumptions across ontologies are also been explained in our method which are not involved in DDL.

\section{RELATED WORK}

Jimenez-Ruiz and Grau[10] base their work on global interpretation . They propose a framework to check and repair consistency which fully makes use of existing ontology debugging technology.

In [3][4], DDL is discussed. Borgida and Serafini introduce distributed description logics to express ontologies connected with ontology mappings which called bridge rules. They use domain relation to interpret bridge rules. Serafini [12][13] continues to design reasoning algorithms of DDL. 
Connected ontology servers as a bridge between local ontology and shard ontology. Grau[9] talks about linking ontologies in E-connections.

Bao[1][2] discusses modular ontologies and its semantics. At some extent, we can conceive of ontology integration as some modular ontologies linked together. But some of its features are not suitable for ontology integration.

Similar to ontology integration, Fahad[8] talks about semantics of ontology merge and algorithms of check consistency. As Flouris[8] have talked about, there are some differences between ontology integration and ontology merge. So ontology integration cannot fully adopt methods proposed in [8].

\section{CONCLUSIONS AND FUTURE WORK}

In this paper, we talk about ontology integration under the background of information integration. The semantics of on-tology integration is our focus. Distributed interpretation not global interpretation is employed to explain its semantics. In our method, a set of interpretations correspond to global on- tology and local ontologies respectively. The relations between global ontology and local ontology are expressed by bridge rules and interpreted with theory came from semantic import. As long as these bridge ruels can be satisfied by distributed interpretation, ontology integration is consistent.

In the future, we will develop al-gorithms of consistency checking based on the ideas given in this paper.

\section{REFERENCES}

[1] Bao, J. Caragea, D. and Honavar, V.G. Modular Ontologies - A Formal Investigation of Semantics and Expressivity. In proc. of ASWC2006, pp.616-631. Springer, Heidelberg. 2006.

[2] Bao, J. Caragea, D., and Honavar, V. A Distributed tableau algorithm for package-based Descrption Logics. In the $2^{\text {nd }}$ International
Workshop On Context Representation And Reasoning(CRR2006), 2006.

[3] Borgida, A. and Serafini, L. Distributed Description Logics: Directed Domain Correspondences in Federated Information Sources. In proc. of CoopIS/DOA/ ODBASE2002, pp.36-52. 2002.

[4] Borgida,A., and Serafini,L. Distributed description logics: Assimilating information form peer sources. Journal on Data Semantics. pp.153-184. 2003.

[5] Chen, H., Wu, Z., Wang, H. and Mao, Y. RDF/RDFS-based Relational Database Integration. In proc. of ICDE2006. 2006.

[6] Dou, D., LePendu, P. Ontology-based Integration for Relational Databases. In proc. of SAC2006, Dijon France, pp.461-466. 2006.

[7] Fahad, M., Moalla, N., Bouras, A. Towards ensuring Satisfiablity of Merged Ontology. Procedia Computer Science, vol4, pp.2216-2225. 2011.

[8] Flouris,G., Manakanatas, D., Kondylakis, H., Plexousakis, D. and Antoniou, G. Ontology change: classification and survey. The Knowledge Engineering Review, vol.23:2, pp.117-152. Cambridge University Press. 2008.

[9] Grau,B.C., Parsia, B. an Evren Sirin. Combining OWL ontologies using E-connections. Journal of Web Semantics, vol.4, pp.40-59. 2006.

[10] Jimernez-Ruiz, E., Grau, B.C., Horrocks, I., and Rafael Berlanga. Ontology Integration Using Mappings: Towards Getting the Right Logical Consequences. In proc. of ESWC2009, pp.173-187. Springer Heidelberg. 2009.

[11] Pan,J.Z., Serafini, L. and Zhao, Y. Semantic Import: An Approach for Partial Ontology Reuse. In $1^{\text {st }}$ International Workshop on Modular Ontologies(WoMo2006), co-located with ISWC2006. 2006.

[12] Serafini,L., Tamilin, A. DRAGO:Distributed Reasoning Architecture for the semantic web. In proc. of ESWC2005, pp.361-376. Springer, Heidelberg. 2005

[13] Serafini, L., Borgida, A. Tamilin, A. Aspects of distributed and modular ontology reasoning. In proc. of IJCAI2005. 2005.

[14] Wache,H., Vogele, T.,Visser, U., Stuckenschmidt, H., Schuster, G, Neumann, H. and Hubner, S. Ontology-based Integration of Information - A survey of existing approaches. In proc. of IJCAI2001, Seattle, WA. pp.108-117. 2001. 